\title{
PROFESSOR ISAAC GOLDHIRSCH 11 October 1949-29 April 2010
}

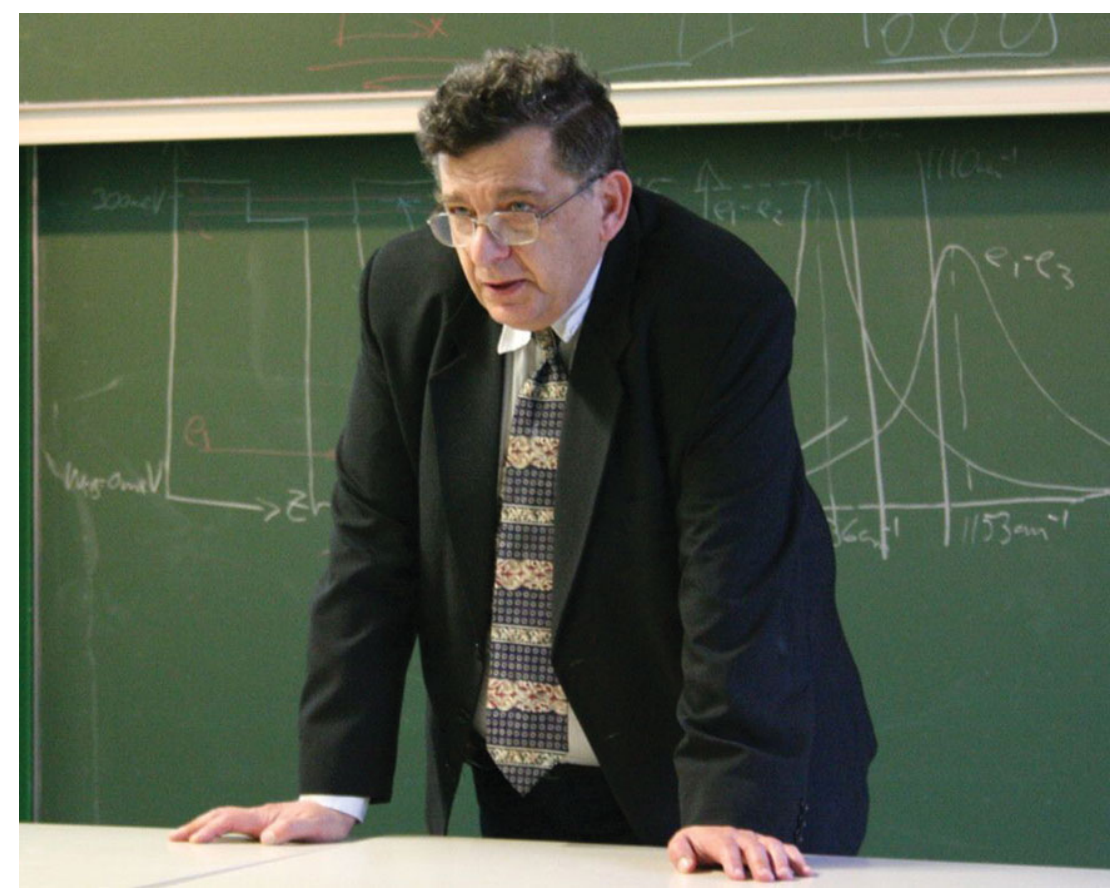

(c) Jonathan Kolmer

Professor Isaac Goldhirsch, the Raquel and Manuel Klachky Chair of Rheological Flows at the School of Mechanical Engineering of Tel-Aviv University, Israel, died unexpectedly on April 29 at age 60 while on sabbatical leave at the University of Erlangen-Nuremberg, Germany.

A dedicated and tireless man of science, Isaac Goldhirsch made important contributions to statistical mechanics, solid-state physics, fluid mechanics, granular physics and applied mathematics. He served as an associate editor of the Journal of Fluid Mechanics, as a founding editor of Granular Matter and was on the editorial board of the Journal of Scientific Computing. Isaac was one of the two Israeli members of the General Assembly for the International Union of Theoretical and Applied Mechanics (IUTAM). He was also a member of the IUTAM Bureau, the organ responsible for executing the resolutions concerning IUTAM that are made by the General Assembly. In particular, Isaac's wisdom and organizational skills were widely recognized.

Isaac was a prolific thinker and author, a decisive editor, an energetic and brilliant collaborator, a dedicated teacher and an entertaining speaker. He enriched and enlivened the meetings and workshops that he attended by employing his vast knowledge of physics and general erudition to illuminate, embellish and question points made by a speaker. He was never at a loss for a new idea or another reference. At conferences, Isaac would be a special draw with many attracted to his 
well-prepared, deep, yet crystal-clear, presentations. As a person, he always showed compassion and respect for others; he had a wry appreciation of human nature, and was an excellent storyteller and a delightful companion. He spoke eight languages fluently.

Isaac Goldhirsch was born in 1949 in Radauti, Romania. His undergraduate degree with special distinction was from the Hebrew University of Jerusalem in physics and mathematics; he received his MSc and PhD from the Weizmann Institute in 1980 in physics and chemical physics, where he won the Feinberg Graduate School's Kennedy Prize. He then spent two important years as a post-doctoral associate with Irwin Oppenheim at the Massachusetts Institute of Technology. Following that, Isaac joined Tel-Aviv University, where he rose through the ranks to hold the Raquel and Manuel Klachky Chair of Rheological Flows.

Along the way, Isaac developed interests in the physics of granular material and it was this subject that occupied him for much of the past 20 years. In his seminal studies of granular materials, Isaac provided the basic theory for inelastic kinetic systems, numerical examples of clustering instabilities and an explanation of their origin; he placed the normal stress differences seen in collisional granular flows in the context of the kinetic theory, and he devised a rational method for coarse-graining granular systems. All these activities have had critical impact on the understanding of the behaviour of the physics of the granular state and its differences from the more classical elastic kinetic systems. Indeed, he described himself as a 'sociologist of particles', reflecting the fact that sociologists strive to comprehend society as a consequence of individual human interactions among their constituent particles. An influential summary of his views on dilute granular systems is given in his Annual Review of Fluid Mechanics article from 2003.

In addition, he was the author of influential reviews and research articles on coarse graining of condensed matter, convection, chaotic dynamics and asymptotic and numerical methods. He made core contributions to studies of dense granular systems, thermophoresis, random walks on networks, accelerated molecular dynamics, stability of fluids, lattice methods for fluids, coarse graining of complex fluids with limited scale separation (especially using a powerful combination of analysis and computation) and the design of large-scale data-storage systems.

He was a devoted husband to Rivka and a father of two adult daughters, Adi and Netta. We shall miss him dearly.

Robert BeHringer, Duke University

James Jenkins, Cornell University

Touvia MiLoh, Tel-Aviv University Steven OrSZaG, Yale University ThORSTEN PösChel, Erlangen-Nuremberg University

Philip Rosenau, Tel-Aviv University Stuart Savage, McGill University Zeev Schuss, Tel-Aviv University Lev Shemer, Tel-Aviv University 